\title{
Assistência e Enfermagem a Indivíduos em Morte Encefálica: Avaliação de Qualidade
} Nursing Assistance for Individuals With Brain Death: A Quality Assessment

\author{
HIASMIN BATISTA RODRIGUES ${ }^{1}$ \\ DENISE LIMA NOGUEIRA ${ }^{2}$ \\ TAMIRES ALEXANDRE FÉLIX ${ }^{3}$ \\ DIÓGENES FARIAS GOMES ${ }^{4}$
}

\section{RESUMO}

Objetivo: Avaliar a qualidade dos cuidados de enfermagem prestados a indivíduos em morte encefálica de um hospital de ensino do Ceará, analisando as anotações, as prescrições e os procedimentos de enfermagem, diante do referencial de padrão de positividade de qualidade. Material e Métodos: Tratou-se de um estudo avaliativo e documental com abordagem quantitativa em que foram analisados 23 prontuários de indivíduos em morte encefálica através de um instrumento adaptado da Assessoria de Controle da Qualidade da Assistência de Enfermagem de um hospital universitário do Paraná, e que, através do padrão de positividade, pôde-se delinear o perfil de preenchimento dos registros de enfermagem da unidade de terapia intensiva em estudo. Resultados: Diante do instrumento utilizado, pôdese identificar que $61 \%$ dos indivíduos em ME eram do sexo masculino com predominância de causa base o trauma crânioencefálico $(56,5 \%)$ e, quando em análise, os registros preenchidos, pôde-se verificar que, em $27,93 \%$ destes, havia ausência de preenchimento, valor superior ao que era considerado limítrofe para bons resultados $(5 \%)$, bem como o valor de preenchimento incompleto que alcançou $20,65 \%$, sendo que o esperado seria até $15 \%$. Conclusão: Identificouse que o quesito não preenchido/ausente esteve superior ao padrão de positividade desejado, situação que atinge a continuidade do cuidado prestado e o respaldo legal da assistência ofertada.

\section{DESCRITORES}

Avaliação em Saúde. Registros de Enfermagem. Morte Encefálica.

\begin{abstract}
Objective: To evaluate the quality of nursing care provided to individuals with brain death admitted to a teaching hospital in the State of Ceará, Brazil. Nursing notes, prescriptions, and procedures were analyzed in face of the referential standard for quality positivity. Material and Methods: This was an evaluative and documentary study with a quantitative approach. A total of 23 medical records from individuals with brain death were analyzed through an instrument adapted from the Nursing Assistance Quality Control Office from an University Hospital in Paraná State. Based on positivity pattern, it was possible to determine the filling profile of the nursing records of the intensive care unit under study. Results: The majority of the individuals with brain death were males $(61 \%)$, with a predominance of cranio-encephalic trauma $(56.5 \%)$ as the underlying cause. Of these, $27.93 \%$ of the records showed no filling, a value higher than what was considered borderline for good results $(5 \%)$, or incomplete filling $(20.65 \%$ - where the expected value should be up to $15 \%$ ). Conclusion: We identified that the unfilled/ missing issue was higher than the desired positivity standard, a situation that affects the continuity of the care provided and the legal support of the assistance.
\end{abstract}

\section{DESCRIPTORS}

Health Evaluation. Nursing Records. Brain Death.

1 Discente lato sensu de Auditoria e Gestão dos Serviços de Saúde pela Faculdade Padre Dourado (FACPED). Integrante da Estratégia Saúde da Familia na Prefeitura Municipal de Uruoca (PMU). Uruoca. Ceará. Brasil.

2 Coordenadora do programa de Residência Multiprofissional em Neonatologia e da Educação Permanente da Santa Casa de Misericórdia de Sobral (SCMS). Sobral. Ceará. Brasil.

3 Docente na Universidade Estadual Vale do Acaraú e Emergencista na Santa Casa de Misericórdia de Sobral (SCMS). Sobral. Ceará. Brasil.

4 Mestrando em Saúde da Família pelo Instituto de Formação Superior do Ceará (IFESC). Preceptor de Educação Permanente na Escola de Formação em Saúde da Família Visconde de Sabóia -EFSFVS. Sobral. Ceará. Brasil. 
$\mathrm{O}$ registros de enfermagem são fatores importantes da Sistematização da Assistência de Enfermagem (SAE) individualizada para o paciente, pois permite a verificação dos procedimentos realizados e possibilita a determinação da qualidade da assistência prestada, atuando como método facilitador do (re)planejamento e da coordenação da assistência, tornando possível modificar o plano de cuidados ofertado, estabelecendo um padrão de qualidade ${ }^{1}$.

Os registros efetuados pela equipe de enfermagem consistem um reflexo de uma assistência ofertada ao paciente, sendo indiscutível a necessidade de estes serem precisos quanto ao que foi praticado e frequentes devido à continuidade do cuidado e ainda devem conter os diagnósticos de enfermagem, os tratamentos e as respostas da assistência médica, assim como as de enfermagem, transparecendo o reflexo da reavaliação e das queixas do paciente quando este está possibilitado de descrevê-las ${ }^{1}$. Destarte, a praticidade e agilidade dos registros ocorrem na forma de anotações ou evoluções de enfermagem, que consistem em registrar dados brutos e observacionais, sendo realizados por auxiliares, técnicos e enfermeiros. A evolução de enfermagem significa um julgamento dos dados de cada indivíduo ao longo do período de trabalho².

Assim, os registros tornaram-se um instrumento de respaldo legal e administrativo, que permite a todos tomar ciência das ações e dos resultados obtidos após a assistência efetivada ${ }^{3}$, podendo ser utilizados como forma de avaliar a qualidade da assistência.

Nessa perspectiva, registrar a assistência acaba por subsidiar uma intercomunicação permanente entre os membros da equipe multiprofissional ${ }^{4}$, principalmente nos cenários críticos do hospital, onde os mínimos detalhes da assistência necessitam ser conhecidos por todos da equipe, e a comunicação oral, muitas vezes, torna-se falha devido à sobrecarga do serviço. Dessa forma, percebe-se a importância de registros completos nos prontuários, haja vista perfazerem uma comunicação escrita nos setores de assistência à saúde. No caso das Unidades de Terapia Intensiva (UTIs), essa necessidade tornase imprescindível, visto que é o local onde se encontra a maior concentração de pacientes críticos, dentre os quais, citam-se os indivíduos com diagnóstico de Morte Encefálica (ME).

A ME é definida por ser a constatação irremediável e irreversível da lesão nervosa e significa a morte clínica, social e legal do indivíduo, não existindo possibilidade de prognóstico de recuperação ${ }^{5}$. No entanto, para a confirmação de morte encefálica em indivíduos adultos, de acordo com a Associação Brasileira de Transplante de Órgãos ${ }^{6}$ devem ser realizados dois exames clínicos e um exame complementar, cujos resultados, para efeito de diagnosticar o indivíduo em morte encefálica, devem ser positivos.

Assim, em um setor com características bem peculiares, o qual reúne um montante de pessoas em estado crítico, além do quantitativo de indivíduos que legalmente estão mortos, há a necessidade de uma avaliação dos registros que são realizados pelos profissionais ali atuantes para que demandem perspectivas aos gestores da unidade, concernentes às práticas ofertadas.

No contexto da qualidade da assistência de enfermagem aos pacientes críticos, questionase: qual é a qualidade dos cuidados de enfermagem proporcionados aos indivíduos em morte encefálica?

Assim, a verificação da qualidade dos serviços que são ofertados tornou-se uma forma imprescindível de fazer o planejamento, a organização, a coordenação e a avaliação das atividades desenvolvidas ${ }^{7}$. E mediante dessa avaliação, podem-se considerar três dimensões, quais sejam: 1) estrutura, entendida como os recursos físicos, humanos, materiais, de equipamentos e financeiros necessários para a assistência à saúde; 2) processo, que se refere às atividades envolvendo profissionais de saúde e usuários, incluindo diagnóstico, tratamento, aspectos éticos de relação profissional, equipe de saúde e paciente; e, 3) resultado, que corresponde ao produto final da assistência prestada, considerando a saúde, satisfação de padrões e expectativas dos usuários ${ }^{8}$.

Dessa forma, analisar a qualidade dos cuidados prestados pela equipe de Enfermagem aos indivíduos em $\mathrm{ME}$, a partir dos registros no prontuário, permite a identificação do padrão de qualidade da assistência prestada e das inconformidades decorrentes dessa assistência, destacando suas possíveis causas e repercussões na manutenção desses indivíduos ${ }^{9}$, contribuindo, assim, não só para o desenvolvimento científico sobre a temática, como também para a reorientação e qualificação das práticas de atenção à saúde da equipe de enfermagem no cuidado a esses indivíduos, repercutindo na manutenção de órgãos viáveis para doação e transplantes, e, 
consequentemente, na qualidade de vida das pessoas que aguardam por doações e transplantes de órgãos ${ }^{10}$.

O objetivo do estudo foi o de avaliar a qualidade dos cuidados de enfermagem prestados a indivíduos em ME em um hospital de ensino do norte do Ceará, analisando as anotações, as prescrições e os procedimentos de enfermagem, diante do referencial de padrão de positividade de qualidade ${ }^{7}$.

\section{MATERIAL E MÉTODOS}

Tratou-se de um estudo avaliativo, exploratório-descritivo e documental, com abordagem quantitativa, para o qual utilizou o referencial de Donabedian, segundo o qual o grau de sucesso das ações relacionadas à saúde, em se autogovernarem, de modo a prevenir a exploração ou a incompetência, tem como objetivo uma monitorização contínua da qualidade para a identificação precoce de desvios dos padrões, a fim de serem corrigidos, e ordena-se na tríade: estrutura, processo e resultado ${ }^{8}$.

No entanto, para fins desse estudo, abordaremos especificamente o componente processo, pois, como reitera o autor supracitado, a avaliação de processo do cuidado é realizada por meio de uma comparação entre os procedimentos empregados e os estabelecidos pelos próprios profissionais de saúde, como normas e protocolos ${ }^{8}$.

O estudo foi realizado na UTI - Bloco Emergência de um hospital de ensino da região norte do Estado do Ceará, visto que este setor possui 10 dos 19 leitos de terapia intensiva destinados a adultos, no hospital em estudo ${ }^{11}$. Teve como período de coleta de dados os meses de abril a junho de 2016.

Também, o local escolhido para o estudo se deu devido sua representatividade para a Região de Saúde, sendo referência para mais de 55 municípios, tendo em sua composição de recursos instalados uma Comissão Intra-Hospitalar de Doação de Órgãos e Tecidos para Transplante (CIHDOTT) e sede da Organização de Procura de Órgãos (OPO) da região.

Foram analisados os prontuários de pacientes admitidos no ano de 2015 no setor supracitado, que continham diagnóstico médico de ME. Os prontuários em que constava apenas a abertura do protocolo de ME (realização do primeiro exame clínico, ou realização de exame complementar sem dados confirmados de $\mathrm{ME}$, devido parada cardiorrespiratória antes da conclusão diagnóstica), ou casos confusos que não reverberavam um diagnóstico consistente, não foram considerados neste estudo.

Os prontuários foram localizados junto ao Serviço de Arquivo Médico e Estatística (SAME), sendo listados 34 prontuários de indivíduos internados com o diagnóstico de ME. Destes, seis tiveram o protocolo de ME iniciado, porém não concluídos devido assistolia antes da finalização dos exames, e cinco prontuários não foram localizados no setor SAME. Destarte, foram incluídos neste estudo um total de 23 prontuários.

Foi aplicado um formulário para coleta de informações junto aos prontuários dos indivíduos, utilizando-se por base o instrumento proposto por Haddad como instrumento da Assessoria de Controle da Qualidade da Assistência de Enfermagem de um hospital universitário do Paraná ${ }^{12}$, o qual foi adaptado e utilizado neste estudo após autorização da autora, respeitando seus direitos autorais. Assim, foram avaliadas as anotações, as prescrições e os procedimentos de enfermagem.

Foram considerados registros de enfermagem todas as anotações realizadas pela equipe de enfermagem, em documento próprio da instituição, nos espaços destinados às anotações referentes aos cuidados prestados, intercorrências, observações e impressões sobre o paciente e/ou acompanhantes, bem como os registros da equipe no espaço destinado à checagem dos procedimentos prescritos.

A análise dos dados foi realizada através da construção de uma planilha no programa Excel $2010^{\circledR}$, com cálculo de frequência simples, e utilizando a análise do padrão de qualidade dos registros de enfermagem compreendendo cinco níveis: 1- não se aplica; 2- completo; 3- incompleto; 4- não preenchido; e 5- incorreto ${ }^{12}$.

O critério "não se aplica" foi utilizado quando os critérios apresentados no instrumento de avaliação não tiveram relação direta com o processo de cuidar da enfermagem em indivíduos em ME. $\mathrm{O}$ quesito "completo" foi utilizado quando todo o critério de preenchimento estabelecido para cada subitem foi satisfeito completamente. Foram classificados como "incompleto", os subitens que atendiam apenas parcialmente os critérios de preenchimento. O critério "não preenchido" foi utilizado quando não houve qualquer anotação correspondente ao subitem descrito. Os registros de enfermagem foram classificados como "incorreto" 
quando havia, pelo menos, uma anotação sobre o subitem abordado, no entanto, com este não atendendo aos critérios de preenchimento correspondente.

Após análise e classificação dos registros, estes foram quantificados e transformados em porcentagens para reclassificação de acordo com o padrão de qualidade de positividade ${ }^{7}$. Assim, foram considerados registros com bons resultados aqueles que atingiram níveis de preenchimento igual ou maior a $80 \%$, não ultrapassando $15 \%$ para o quesito incompleto, $5 \%$ para o não preenchido e $0 \%$ incorretos.

Para tanto, a pesquisa somente teve início após a anuência do fiel depositário das informações dos prontuários dos indivíduos. Foi também apresentado ao setor SAME, o Termo de Compromisso de Utilização dos Dados, a fim de firmar os compromissos éticos estabelecidos pelos princípios da autonomia, da não malevolência, da beneficência e da justiça.

Neste contexto, as informações coletadas, foram utilizadas especificamente para fins deste estudo, atendendo aos princípios éticos propostos na Resolução 466/12, por meio do qual foi respeitada a confidencialidade, a privacidade e a proteção da imagem, garantindo a não utilização das informações em prejuízo das pessoas e/ou das comunidades $^{13}$.

O projeto foi submetido ao Departamento de Ensino, Pesquisa e Extensão do hospital em estudo para a obtenção da carta de anuência, e, posteriormente ao Comitê de Ética em Pesquisa com Seres Humanos da Universidade Estadual Vale do Acaraú, recebendo parecer favorável sob o $\mathrm{n}^{\circ}$ 1.485.208/2016.

\section{RESULTADOS}

Foram identificadas características dos indivíduos durante a análise dos prontuários. O sexo masculino atingiu $61 \%(n=14)$ e o sexo feminino $39 \%(n=9)$. Obteve-se, ainda, um somatório total de dias de internamento das 23 pessoas hospitalizadas igual a 89 dias. Assim, estabeleceuse uma média de 3,86 dias de internação e idade média de 34,78 anos.

Diante das causas base de ME houve uma predominância de traumatismo crânio-encefálico grave em 56,5\% ( $n=13)$ dos casos, o acidente vascular encefálico em $34,8 \%(n=8)$, e em $8,7 \%$ $(n=2)$ tumor intracraniano.

Igualmente, através do padrão de qualidade de positividade ${ }^{7}$, pode-se delinear o perfil de preenchimento dos registros de enfermagem da unidade em estudo (Figura 1), através dos quesitos estabelecidos no instrumento utilizado na Assessoria de Controle da Qualidade da Assistência de Enfermagem ${ }^{12}$, para avaliar a qualidade dos registros de enfermagem.

As Tabelas 1, 2 e 3 a seguir apresentam os percentuais encontrados referentes aos itens de

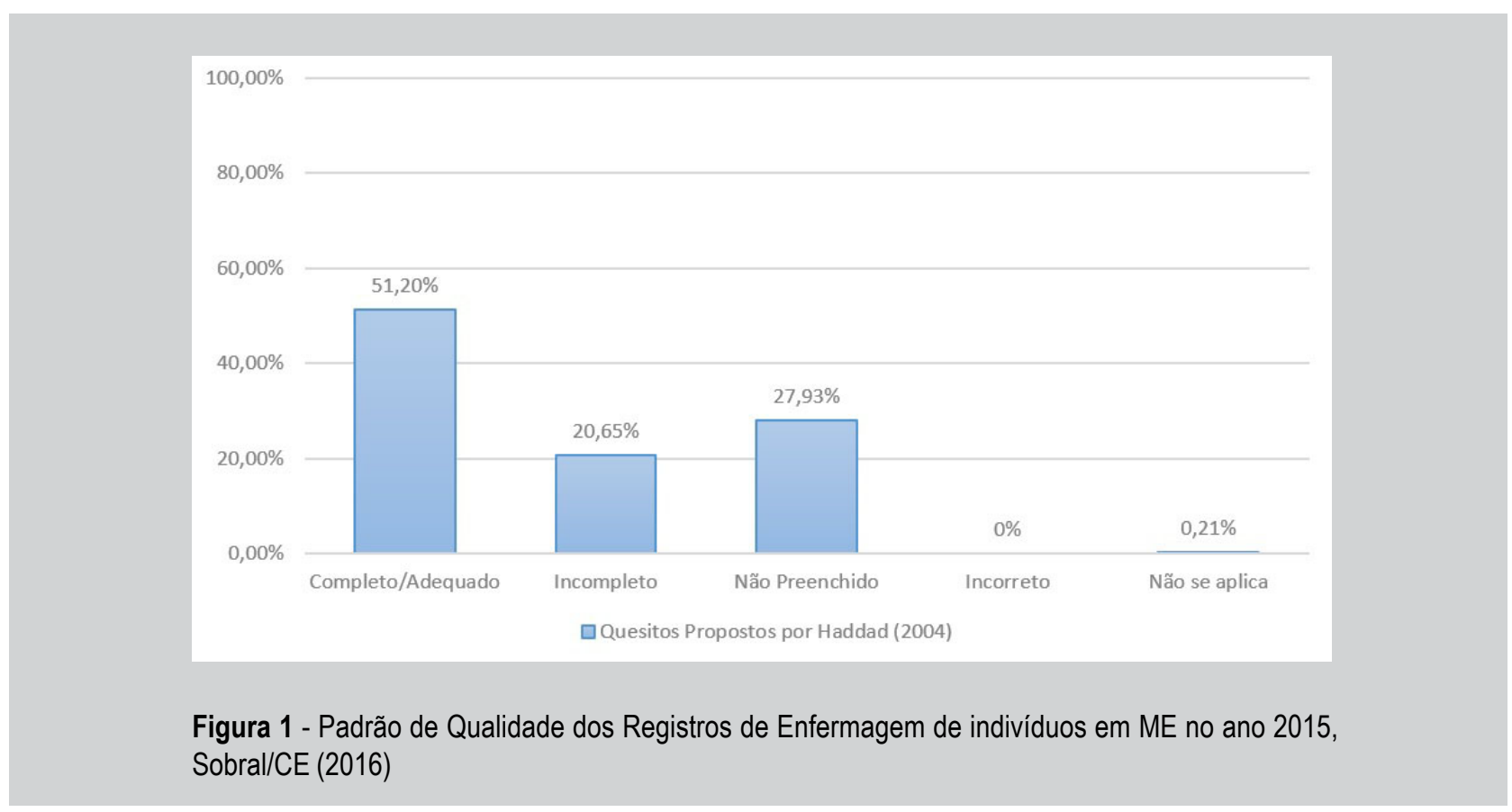


análise de acordo com o instrumento utilizado, onde foram analisados respectivamente: as anotações de enfermagem, as prescrições de enfermagem e os procedimentos de enfermagem.

Verifica-se na Tabela 1 que apenas os subitens que analisaram as anotações referentes às respostas da prescrição de enfermagem e os registros referentes ao débito urinário atingiram padrão satisfatório de qualidade da assistência de enfermagem e que não foram encontrados preenchimentos incorretos, embora algumas tenham sido classificadas como incompletas.

A Tabela 3 apresenta os percentuais encontrados referentes à realização dos procedimentos de enfermagem, os quais estavam inclusos nas prescrições de enfermagem e aprazados, que eram atuações rotineiras em cada plantão da equipe de enfermagem. No entanto, constatou-se que somente o subitem que analisava a realização de sinais vitais no mínimo três vezes ao dia, atendeu ao padrão de qualidade da assistência de enfermagem.

\begin{tabular}{|c|c|c|c|c|c|}
\hline Itens Avaliados & & & Critérios & & \\
\hline $\begin{array}{c}\text { ANOTAÇÕES DE } \\
\text { ENFERMAGEM }\end{array}$ & $\begin{array}{l}\text { Não se } \\
\text { Aplica }\end{array}$ & Completo & Incompleto & $\begin{array}{l}\text { Não } \\
\text { Preenchido }\end{array}$ & Incorreto \\
\hline $\begin{array}{l}\text { Há pelo menos uma anotação } \\
\text { descritiva em cada plantão? }\end{array}$ & $0 \%$ & $19 \%$ & $70 \%$ & $11 \%$ & $0 \%$ \\
\hline $\begin{array}{l}\text { Há horário e rubrica em cada } \\
\text { anotação? }\end{array}$ & $0 \%$ & $44 \%$ & $54 \%$ & $2 \%$ & $0 \%$ \\
\hline $\begin{array}{l}\text { As anotações correspondem } \\
\text { aos itens da prescricão? }\end{array}$ & $0 \%$ & $99 \%$ & $0 \%$ & $1 \%$ & $0 \%$ \\
\hline $\begin{array}{l}\text { As anotações } \\
\text { observação de sinciam } \\
\text { sintomas? }\end{array}$ & $0 \%$ & $11 \%$ & $78 \%$ & $11 \%$ & $0 \%$ \\
\hline $\begin{array}{l}\text { Existe uma anotação } \\
\text { descritiva de admissão, } \\
\text { transferência, alta ou óbito. }\end{array}$ & $0 \%$ & $37 \%$ & $3 \%$ & $60 \%$ & $0 \%$ \\
\hline $\begin{array}{l}\text { Há anotações referentes às } \\
\text { intercorrências? }\end{array}$ & $0 \%$ & $1 \%$ & $1 \%$ & $98 \%$ & $0 \%$ \\
\hline $\begin{array}{l}\text { Há anotações sobre o aspecto } \\
\text { e evolução de lesões } \\
\text { cutâneas? }\end{array}$ & $0 \%$ & $3 \%$ & $0 \%$ & $97 \%$ & $0 \%$ \\
\hline $\begin{array}{l}\text { Há anotações sobre o débito } \\
\text { urinário? }\end{array}$ & $0 \%$ & $95 \%$ & $5 \%$ & $0 \%$ & $0 \%$ \\
\hline
\end{tabular}

\begin{tabular}{|c|c|c|c|c|c|}
\hline Itens Avaliados & \multicolumn{5}{|c|}{ Critérios } \\
\hline $\begin{array}{ll}\text { PRESCRIÇÃO } & \text { DE } \\
\text { ENFERMAGEM } & \end{array}$ & $\begin{array}{l}\text { Não se } \\
\text { Aplica }\end{array}$ & Completo & Incompleto & $\begin{array}{l}\text { Não } \\
\text { Preenchido }\end{array}$ & Incorreto \\
\hline $\begin{array}{l}\text { Há pelo menos uma } \\
\text { prescrição diária para o } \\
\text { indivíduo? }\end{array}$ & $0 \%$ & $75 \%$ & $1 \%$ & $24 \%$ & $0 \%$ \\
\hline $\begin{array}{l}\text { A prescrição indica } \\
\text { cuidado de enfermagem } \\
\text { especial (individual) de } \\
\text { acordo com a patologia e } \\
\text { necessidade? }\end{array}$ & $0 \%$ & $79 \%$ & $0 \%$ & $21 \%$ & $0 \%$ \\
\hline
\end{tabular}




\begin{tabular}{|c|c|c|c|c|c|}
\hline \multirow{2}{*}{$\begin{array}{l}\text { Itens Avaliados } \\
\text { PROCEDIMENTOS DE } \\
\text { ENFERMAGEM }\end{array}$} & \multicolumn{5}{|c|}{ Critérios } \\
\hline & $\begin{array}{l}\text { Não se } \\
\text { Aplica }\end{array}$ & Completo & Incompleto & $\begin{array}{c}\text { Não } \\
\text { Preenchido }\end{array}$ & Incorreto \\
\hline $\begin{array}{l}\text { Há registros de higiene } \\
\text { corporal diária? }\end{array}$ & $0 \%$ & $69 \%$ & $24 \%$ & $7 \%$ & $0 \%$ \\
\hline $\begin{array}{l}\text { Há registros de sinais } \\
\text { vitais, pelo menos três } \\
\text { vezes ao dia? }\end{array}$ & $0 \%$ & $97 \%$ & $1 \%$ & $2 \%$ & $0 \%$ \\
\hline $\begin{array}{l}\text { Há registro da localização } \\
\text { dos } \quad \text { procedimentos } \\
\text { invasivos? }\end{array}$ & $0 \%$ & $2 \%$ & $1 \%$ & $97 \%$ & $0 \%$ \\
\hline $\begin{array}{l}\text { Há registro da realização } \\
\text { do exame físico, pelo } \\
\text { menos uma vez por } \\
\text { período? }\end{array}$ & $0 \%$ & $18 \%$ & $75 \%$ & $7 \%$ & $0 \%$ \\
\hline $\begin{array}{l}\text { Há registro de PVC, pelo } \\
\text { menos uma vez por } \\
\text { periodo? }\end{array}$ & $4 \%$ & $20 \%$ & $28 \%$ & $48 \%$ & $0 \%$ \\
\hline $\begin{array}{l}\text { Há registro de aspirações } \\
\text { orais, nasotraqueais, tubo } \\
\text { orotraqueal } \\
\text { traqueóstomo? }\end{array}$ & $0 \%$ & $10 \%$ & $15 \%$ & $75 \%$ & $0 \%$ \\
\hline $\begin{array}{l}\text { Há registro de mudança de } \\
\text { decúbito proqramada? }\end{array}$ & $0 \%$ & $77 \%$ & $8 \%$ & $15 \%$ & $0 \%$ \\
\hline $\begin{array}{l}\text { Há registro de hidratação } \\
\text { ocular? }\end{array}$ & $0 \%$ & $81 \%$ & $9 \%$ & $10 \%$ & $0 \%$ \\
\hline
\end{tabular}

\section{DISCUSSÃO}

A avaliação de processo possibilitou a identificação do perfil da assistência de enfermagem, a qual, de acordo com os resultados, mostrou insuficiente preenchimento dos registros de enfermagem, atingindo $27,93 \%$, valor acima da porcentagem esperada. Destes, $44 \%$ não possuíam a identificação do profissional ou horário da anotação, o que evidencia uma característica insatisfatória para continuidade do cuidado na equipe de enfermagem e multiprofissional.

Os registros de enfermagem pressupõem todo o conhecimento possível acerca das condições de saúde dos indivíduos em sua responsabilidade e são fonte de pesquisa multiprofissional ${ }^{14}$. Assim, todo o processo praticado fica legalmente respaldado, e torna possível avaliar as práticas profissionais, possibilitando um mapeamento situacional.

Perante a ambiência hospitalar, todos os sujeitos são susceptíveis a qualquer instabilidade do organismo, e, nesse aspecto, houve uma deficiência nas anotações relacionadas às intercorrências, visto que, em $98 \%$ dos registros realizados no período de internação, não houve preenchimento relacionado, assim como em $97 \%$ não apresentavam registros relacionados às lesões cutâneas existentes, ambos superiores ao padrão de até $5 \%$ de não preenchimento ${ }^{7}$. Com relação às lesões cutâneas, leva-se em consideração que 56,5 $\%(n=13)$ dos indivíduos que evoluíram para ME tinham como diagnóstico inicial de traumatismo crânio-encefálico grave e que, havia anotações em $15,3 \%(n=2)$ destes, relatando trocas de curativos, mas sem os aspectos das lesões e materiais utilizados em sua troca, dados estes referentes à realização de curativos, fazendo parte da atuação do enfermeiro ${ }^{15}$.

O registro de débito urinário é parte rotineira dos plantonistas da equipe do setor, sendo realizado no mínimo uma vez por plantão, alcançando um padrão de $95 \%$ de registro completo e somente $5 \%$ de forma incompleta, bem como o registro de sinais vitais ao mínimo de três vezes por dia, 
transparecendo eficácia em registro e realização pela equipe.

Diante da complexidade de ofertar uma assistência de qualidade o uso de um instrumento facilitador da aplicabilidade do processo permite o alcance de resultados pelos quais o enfermeiro é responsável ${ }^{16}$, utilizando, dessa forma, a SAE, que é composta de etapas para sua formulação completa, trazendo como parte essencial do processo construtivo a prescrição de enfermagem. No entanto, em somente $75 \%$ dos registros havia uma prescrição de enfermagem diária, indicando a falta de reavaliação do estado do indivíduo, cuja ausência de registros compromete a propriedade dos cuidados devido ser considerado fator impeditivo para a reavaliação situacional dos indivíduos.

A higienização dos sujeitos faz parte das necessidades humanas básicas de todo ser e que não requer profundo conhecimento científico e nem muita habilidade ${ }^{17}$. Conquanto, no presente estudo, somente em $69 \%$ dos registros foi verificada completa realização deste cuidado, inferindo uma realização parcial ou a não realização destes cuidados de higiene. Durante o período de internação, ocorre a visitação de familiares e entes, ou seja, existe a necessidade de bem-estar psicoemocional destes, e frente à ausência de higiene adequada do indivíduo em ME, pode haver uma influência negativa neste bem-estar.

A PVC reflete a pressão de enchimento do átrio direito, propiciando informações sobre o volume sanguíneo intravascular, além da função ventricular direita $^{18}$, influenciando em decisões sistêmicas. Assim, diante dos achados, não houve o alcance do padrão de qualidade, sendo que, em apenas $4 \%$ dos registros foi identificada a não possibilidade de verificação da PVC, visto que estava descrito nos achados, que o indivíduo possuía apenas acesso venoso periférico.

O fato de os sujeitos do estudo estarem em ME faz subtender que há a impossibilidade de respiração espontânea dos indivíduos. Logo, todos se encontravam com vias aéreas avançadas e consequente ventilação mecânica. Visto que, no cenário de estudo tem-se o profissional fisioterapeuta somente no período diurno, infere-se que, durante o período de 12 horas noturnas, o enfermeiro-quem fica responsável por aspirar as secreções de vias aéreas. No entanto, houve um percentual de $75 \%$, sem qualquer anotação referente à realização deste procedimento, e em somente $10 \%$, havia relatos sobre a quantidade e o aspecto das secreções.

A falta de mobilidade dos indivíduos no leito reforça a dificuldade de manutenção da integridade da pele, contudo, a mudança de decúbito, que previne este agravo foi completamente checada em apenas $77 \%$ dos registros, todavia esse percentual foi superior ao encontrado em outros estudos ${ }^{19}$.

Posto que, diante da ambientação de terapia intensiva, os olhos podem ficar ressecados devido à fragilidade do tecido corneano, estes necessitam de hidratação frequente. Assim, durante verificação dos registros de hidratação ocular, $81 \%$ dos registros nos prontuários estavam completos, mas obtendo $10 \%$ de registro ausentes, o que não se adequa ao padrão de positividade ${ }^{7}$.

Perante os percentuais encontrados correlacionados ao padrão de positividade estabelecido $^{7}$, pode-se inferir uma desinformação dos profissionais no cumprimento que rege o exercício profissional ${ }^{20}$, que dispõe sobre o registro das ações no prontuário do paciente, e em documentos próprios da enfermagem, refletindo o engajamento que a equipe possui durante a oferta assistencial, e permitindo a disseminação de informações.

Outro padrão para o índice de positividade ${ }^{19}$ para classificar a assistência prestada pode ser utilizado para comparação dos achados, mas com os quantitativos aqui encontrados, em sua maioria, os registros se classificam como padrão de qualidade sofrível ou limítrofe em sua aplicabilidade.

Diante dos resultados encontrados, podese depreender uma não compreensão por parte dos profissionais, quanto ao objetivo e importância dos registros diante de uma assistência completa, ao enfermeiro compete a supervisão destas práticas e preenchimentos referentes, exemplificando para a equipe uma prática completa dos cuidados, com execução de reavaliações e intervenções sistemáticas nos pacientes.

Apesar de se verificar que a SAE era aplicada, esta não atingia os padrões de positividade almejados. Assim, perante os achados, pode-se afirmar que a assistência de enfermagem prestada a indivíduos em ME necessita de maior atenção e envolvimento profissional, visto que todos que se encontram internados devem permanecer sob ótica dos cuidados multiprofissionais até a ausência de batimentos cardíacos.

No entanto, este estudo apresentou como forma de limitação o fato de avaliar apenas indivíduos em ME no setor de terapia intensiva do bloco emergência do hospital cenário, configurando-se em uma avaliação relativa da qualidade da assistência no hospital em estudo, sendo necessário o desenvolvimento de outros estudos avaliativos nas demais UTI's do referido hospital. 


\section{CONCLUSÃO}

Dentre os principais achados deste estudo, identificou-se que o quesito não preenchido/ausente esteve superior ao padrão de positividade desejado, situação que atinge a continuidade do cuidado prestado e o respaldo legal da assistência ofertada, inferindo uma negligência dos profissionais envolvidos.

Diante do exposto, o diagnóstico situacional da qualidade assistencial de enfermagem ao indivíduo em ME, evidencia a necessidade de um aperfeiçoamento da oferta de cuidados, através de um comprometimento da equipe com o ato de registrar para maiores qualificações dos serviços ofertados no setor saúde.

\section{REFERÊNCIAS}

1. Vituri DW, Matsuda LM. Os registros de enfermagem como indicadores da qualidade do cuidado: um estudo documental, descritivo exploratório e retrospectivo. Online Braz J Nurs 2008; 7(1).

2. Santos MGPS, Medeiros MMR, Gomes FQC, Enders BC. Percepção de enfermeiros sobre o processo de enfermagem: uma integração de estudos qualitativos. Rev RENE 2012;13(3):712-23.

3. Zanei SSV, Kawamura MM, Mori S, Cohrs CR, Whitaker IY. Valoração e registros sobre higiene oral de pacientes intubados nas unidades de terapia intensiva. Rev. Min Enferm. 2016; 20.

4. Silva JA, Grossi ACM, Haddad MCL, Marcon SS Avaliação da qualidade das anotações de enfermagem em unidade semi-intensiva. Esc. Anna Nery 2012; 16(3): 576-81.

5. Paraná. Secretaria de Estado da Saúde. Superintendência de Gestão de Sistemas de Saúde. Manual Para Notificação, Diagnóstico De Morte Encefálica E Manutenção Do Potencial Doador De Órgãos E Tecidos/Central Estadual de Transplantes. Curitiba: CET/PR, 2014. Disponível em/Available in: http:/ /www.saude.pr.gov.br/arquivos/File/CET/ Manual_UTI.pdf. Acesso em/Access in: 23 out 2015.

6. Associação Brasileira de Transplantes de Órgãos. 2011. Disponível em/Available in: http://www.abto.org.br/ $\begin{array}{llllllll}a & b & t & 0 & v & 0 & 3 & \text { l }\end{array}$ default.aspx?mn=472\&c=915\&s=0\&friendly=entendendoa-morte-encefalica. Acesso em/Access in: 23 out 2015.

7. Cianciarullo TI, Fugulin FMT, Andreoni S. C \& Q: a hemodiálise em questão: opção pela qualidade assistencial. São Paulo: Ícone; 1998.

8. Donabedian A. The methods and findings os quality assessment and monitoring: an illustraded analysis. Michigan: Health Administration Press; 1985.

9. Dias TCL, Santos JLG, Cordenuzzi OCP, Prochnow AG. Auditoria em enfermagem: revisão sistemática da literatura. Rev. bras. enferm. 2011; 64(5):931-37.

10. Versa GLGS, Murassaki AY, Silva LG, Vituri DW, Mello WA, Matsuda LM. Avaliação da qualidade das prescrições de enfermagem em hospitais de ensino público. Rev Gaúcha Enferm. 2012; 33(2):28-35.

11. BRASIL. Cadastro Nacional dos Estabelecimentos de Saúde. 2016. Disponível em/Available in: http:// cnes.datasus.gov.br/pages/estabelecimentos/ficha/ hospitalar/2312903021114. Acesso em/Access in: 04 ago 2016

12. Haddad MCL. Qualidade da assistência de enfermagem - O processo de avaliação em hospital Universitário Público [Tese de Doutorado]. Ribeirão Preto (SP): Escola de Enfermagem de Ribeirão Preto da Universidade de São Paulo; 2004. 250p.
13. BRASIL. Conselho Nacional de Saúde. Resolução No 466/12. Brasília, DF; 2012. Disponível em/Available in: http://www.conselho.saude.gov.br. Acesso em/ Access in: 28 nov 2015.

14. Colaço A, Menezes FR, Nascimento ERP, Lazzari DD, Böes AA, Jung W. Registro da avaliação de enfermagem em terapia intensiva: discurso do sujeito coletivo. Rev. Enferm. UFSM. 2015; 5(2):257-66.

15. Conselho Federal de Enfermagem [homepage] Resolução n. 0501 de 09 de dezembro de 2015. Regulamenta a competência da equipe de enfermagem no cuidado às feridas e dá outras providências. Disponivel em/ Available in: http://www.cofen.gov.br/ resolucao-cofen-no-05012015_36999.html. Acesso em/ Access in: 02 ago 2016.

16. Conselho Federal de Enfermagem [homepage]. Resolução n. 358 de 15 de outubro de 2009. Dispõe sobre a Sistematização da Assistência de Enfermagem e a implementação do Processo de Enfermagem em ambientes, públicos ou privados, em que ocorre o cuidado profissional de enfermagem, e dá outras providências.

17. Padilha EF, Matsuda LM. Qualidade dos cuidados de enfermagem em terapia intensiva: avaliação por meio de auditoria operacional. Rev Bras Enferm. 2011; 64(4):684-91.

18. Lílian C. Rotina de Monitorização de Pressão Venosa Central. Grupo Hospitalar Conceição. 2010. Disponível em/ Available in: http://www3.ghc.com.br/PROT/ EnfHCR/files/Rotina\%20PVC.pdf. Acesso em/ Access in: 14 jun 2016.

19. Silva RB, Loureiro MDR, Frota OP, Ortega FB, Ferraz CCB. Qualidade da assistência de enfermagem em unidade de terapia intensiva de um hospital escola. Rev Gaúcha Enferm. 2013; 34(4):114-20.

20. Conselho Federal de Enfermagem [homepage]. Resolução n.191 de 31 de maio de 1996. Dispõe sobre a forma de anotação e o uso do número de inscrição ou da autorização, pelo pessoal de Enfermagem, 1996. Disponivel em/Available in: http://www.cofen.gov.br/ resoluo-cofen-1911996-revogou-resoluo-cofen1751994_4250.html. Acesso em/ Access in: 20 jun 2016.

\section{CORRESPONDÊNCIA}

Nome completo: Hiasmin Batista Rodrigues

Endereço: Maestro Pedro Peixoto n. 90, Centro.

CEP: $62460-000$

Uruoca, Ceará, Brasil,

E-mail: hiasmin.6@gmail.com 